\title{
|||||||||||||||||||||||||||||||||||||||||||||||||||||||||||||||||.
}

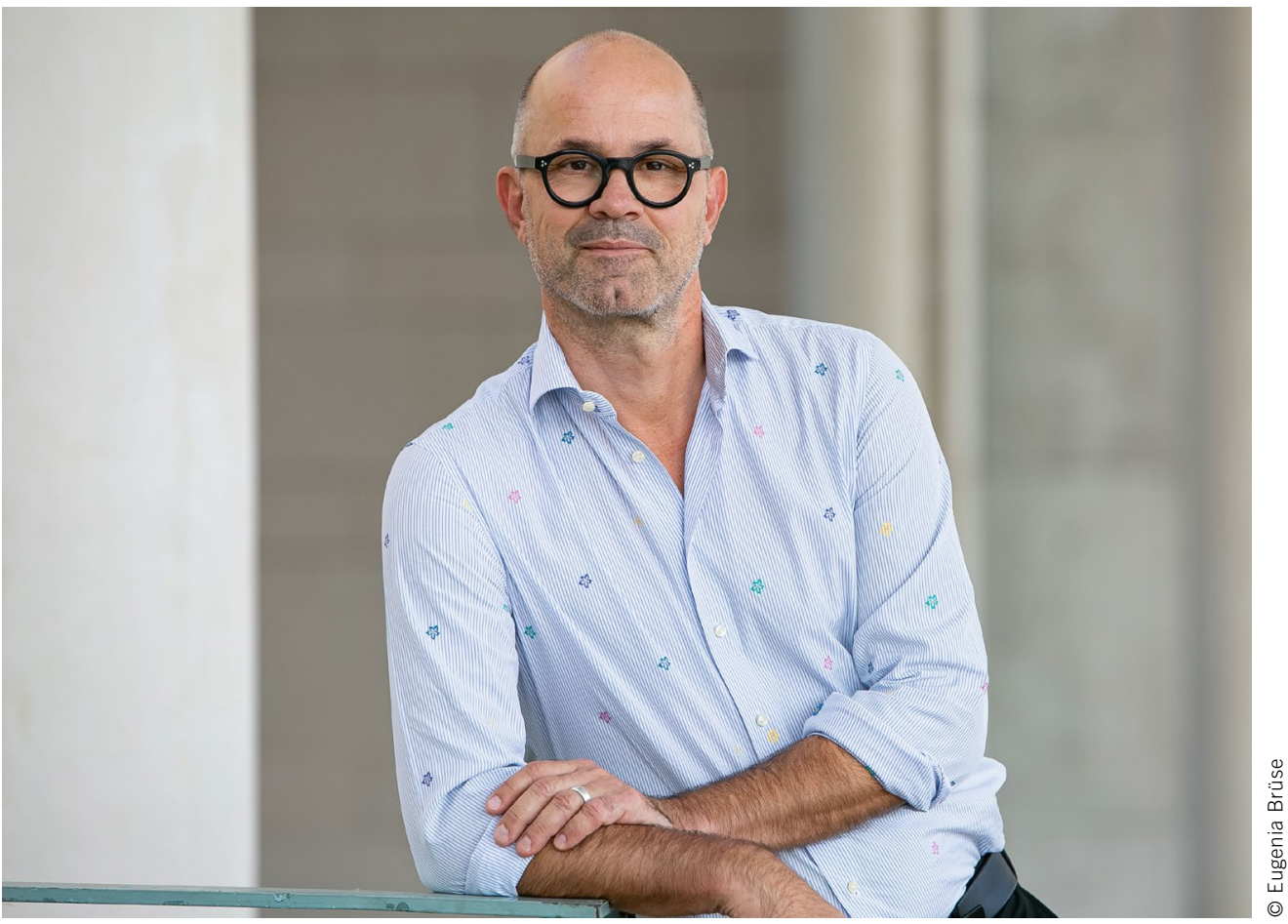

Markus Sigismund

Stellvertretender Referatsleiter im Bereich Prognosen, Statistik und Sondererhebungen des Bundesministeriums für Verkehr und digitale Infrastruktur (BMVI)

\section{Mobilität - Big Data alleine nicht groß}

Zu Fuß, mit dem Fahrrad, mit Bus, Bahn oder dem Auto - jeden Tag legen die Menschen in Deutschland mehr als drei Milliarden Kilometer zurück. Wie können wir die Infrastruktur so gestalten, dass es möglich ist, unsere Wege sicher, schnell und ressourcenschonend zurückzulegen? Ein Schlüssel dafür ist, das Mobilitätsverhalten möglichst gut $\mathrm{zu}$ verstehen.

Die unterschiedlichen Strukturen und Entwicklungen der Mobilität zwischen Stadt und Land, aber auch innerhalb der Städte und der ländlichen Räume stellen eine große Herausforderung dar. Altbekannte Muster lösen sich weiter auf, zum Beispiel konzentrieren sich Arbeitsplätze und Einkaufsmöglichkeiten schon länger nicht mehr im Stadtzentrum. Immer mehr der zurückgelegten Wege verlaufen dispers im Raum. Ein zentrales Hindernis in der Mobilitätsforschung ist das Fehlen geeigneter Daten von Verkehrsverflechtungen in einer kleinräumigen Auflösung. Wir wissen nicht: Wie viele Personen bewegen sich tatsächlich von A nach B?

Im Zeitalter der Digitalisierung drängt sich die Nutzung von Floating Car Data (FCD) und Mobilfunkdaten auf, denn nach zuverlässiger Anonymisierung lassen sich typische Mobilitätsstrukturen auch kleinräumig herleiten. Immer häufiger wird daher gefordert, „klassische“ Befragungen durch „Big Data“ zu ersetzen. Ein solch schlichter Paradigmenwechsel wäre aber vorschnell. Verbunden mit der raschen Verfügbarkeit hatten die Mobilfunkdaten während der Corona-Pandemie für viele Frage- stellungen einen hohen Mehrwert. Für repräsentative Rückschlüsse auf Niveau und Struktur der Mobilität erreichen diese jedoch bisher nicht die erforderliche Qualität. Denn die Daten sind aufgrund der spezifischen Kunden- und Datenstruktur „schief“, also nicht repräsentativ. So sind zum Beispiel kurze Wege bei Mobilfunkdaten untererfasst, denn diese werden innerhalb einer Funkzelle nicht präzise abgebildet. FCD hingegen decken nur den Ausschnitt der Kraftfahrzeuge ab. Und die Daten sagen kaum etwas zu den Zwecken aus, zu denen die Menschen unterwegs sind - an einem für die Prognose der Mobilität wichtigen Punkt wären wir damit nahezu blind.

In einem aktuellen Forschungsprojekt mit dem Akronym „VerBindungen“ führen wir deshalb gemeinsam mit dem Statistischen Bundesamt und weiteren Forschungspartnern die „alte“ und „neue“ Datenwelt aufwendig zusammen: Wir eichen FCD und Mobilfunkdaten an klassischen Datenquellen und werden aus der Synthese belastbare Daten und neue Methodenkenntnisse ableiten. Hierzu können wir auf erste kleinräumige Auswertungen der Pendlerdaten der Bundesagentur für Arbeit, Mikrodaten der Statistischen Ämter des Bundes und der Länder und eigene Erhebungen des BMVI zurückgreifen. Die bisherigen Erfahrungen zeigen: Der Aufwand lohnt sich. Und die klassischen Daten der amtlichen Statistik und ein Kern von Befragungen bleiben unerlässlich, auch um das Potenzial von Big Data groß zu machen. 